\title{
Statistical mechanics of the fluctuating lattice Boltzmann equation
}

\author{
Burkhard Dünweg and Ulf D. Schiller \\ Max Planck Institute for Polymer Research, Ackermannweg 10, D-55128 Mainz, Germany \\ Anthony J. C. Ladd \\ Max Planck Institute for Polymer Research, Ackermannweg 10, D-55128 Mainz, Germany and Chemical Engineering Department, \\ University of Florida, Gainesville, Florida 32611-6005, USA \\ (Received 11 July 2007; published 12 September 2007)
}

\begin{abstract}
We propose a derivation of the fluctuating lattice Boltzmann equation that is consistent with both equilibrium statistical mechanics and fluctuating hydrodynamics. The formalism is based on a generalized lattice-gas model, with each velocity direction occupied by many particles. We show that the most probable state of this model corresponds to the usual equilibrium distribution of the lattice Boltzmann equation. Thermal fluctuations about this equilibrium are controlled by the mean number of particles at a lattice site. Stochastic collision rules are described by a Monte Carlo process satisfying detailed balance. This allows for a straightforward derivation of discrete Langevin equations for the fluctuating modes. It is shown that all nonconserved modes should be thermalized, as first pointed out by Adhikari et al. [Europhys. Lett. 71, 473 (2005)]; any other choice violates the condition of detailed balance. A Chapman-Enskog analysis is used to derive the equations of fluctuating hydrodynamics on large length and time scales; the level of fluctuations is shown to be thermodynamically consistent with the equation of state of an isothermal, ideal gas. We believe this formalism will be useful in developing new algorithms for thermal and multiphase flows.
\end{abstract}

DOI: 10.1103/PhysRevE.76.036704

PACS number(s): 47.11.Qr, 47.57.-s

\section{INTRODUCTION}

Lattice Boltzmann (LB) methods [1,2] have become a popular tool for simulating hydrodynamics, particularly in complex geometries. The underlying model is a regular lattice of sites $\vec{r}$, combined with a small set of velocity vectors $\vec{c}_{i}$, which, within one time step $h$, connect a given site with some of its neighbors. The set of velocities is chosen to be compatible with the symmetry of the lattice. The basic dynamical variables are real-valued populations $n_{i}$; in the present paper, we will consider $n_{i}$ as the mass density associated with the velocity $\vec{c}_{i}$. The LB algorithm is then described by the update rule

$$
n_{i}\left(\vec{r}+\vec{c}_{i} h, t+h\right)=n_{i}^{\star}(\vec{r}, t)=n_{i}(\vec{r}, t)+\Delta_{i}\left\{n_{i}(\vec{r}, t)\right\},
$$

where $\left\{n_{i}\right\}$ denotes the complete set of populations. The $\left\{n_{i}(\vec{r}, t)\right\}$ at each site are first rearranged in a "collision" step, described by $\Delta_{i}$, and then propagated along their respective links. The hydrodynamic fields, mass density

$$
\rho(\vec{r}, t)=\sum_{i} n_{i}(\vec{r}, t)
$$

and momentum density

$$
\vec{j}(\vec{r}, t)=\sum_{i} n_{i}(\vec{r}, t) \vec{c}_{i}
$$

are moments of the discrete velocity distribution $n_{i}(\vec{r}, t)$, while the fluid velocity is given by

$$
\vec{u}(\vec{r}, t)=\vec{j}(\vec{r}, t) / \rho(\vec{r}, t) .
$$

The collisions conserve mass and momentum, hence

$$
\sum_{i} \Delta_{i}=\sum_{i} \Delta_{i} \vec{c}_{i}=0
$$

The algorithm thus satisfies important requirements for simulating hydrodynamic flows - mass and momentum conservation, and locality-but lacks Galilean invariance due to the finite number of velocities. Full rotational symmetry is also lost, but by a suitable choice of velocity set, isotropic momentum transport can be recovered on sufficiently large (hydrodynamic) length scales. Nevertheless, the finite number of velocities always confines the method to flows with small Mach number $u / c_{s} \ll 1$. The speed of sound $c_{s}$ is of order $b / h$, where $b$ is the lattice spacing, or of order $\left|\vec{c}_{i}\right|$.

Most of the LB literature deals with deterministic collision rules, with $\Delta_{i}$ describing a linear relaxation of the distribution $\left\{n_{i}\right\}$ toward the local equilibrium $[3,4]$ :

$$
n_{i}^{e q}(\rho, \vec{u})=\rho a^{c_{i}}\left(1+\frac{\vec{u} \cdot \vec{c}_{i}}{c_{s}^{2}}+\frac{\left(\vec{u} \cdot \vec{c}_{i}\right)^{2}}{2 c_{s}^{4}}-\frac{u^{2}}{2 c_{s}^{2}}\right),
$$

where $a^{c_{i}}>0$ is the weight associated with the speed $\left|\vec{c}_{i}\right|$. The viscosity of the LB fluid is determined by the choice of relaxation rates.

However, to simulate Brownian motion of suspended particles, thermal fluctuations must be included. At the hydrodynamic level, this means adding uncorrelated noise to the fluid stress tensor [5]. In Refs. [6-8] an analogous fluctuating LB model was introduced by making $\Delta_{i}$ a stochastic variable, but in such a way that the noise was only applied to the modes (linear combinations of $\left\{n_{i}\right\}$ ) related to the viscous stress tensor 


$$
\Pi_{\alpha \beta}^{n e q}=\sum_{i} n_{i}^{n e q} c_{i \alpha} c_{i \beta}
$$

here $\alpha$ and $\beta$ denote Cartesian components and $n_{i}^{\text {neq }}=n_{i}$ $-n_{i}^{e q}$ is the nonequilibrium distribution. Although this procedure is correct in the hydrodynamic limit [7,9], it provides poor thermalization on smaller length scales, as was first observed by Adhikari et al. [10]. They introduced a thermalization procedure which applies to all nonconserved modes, with significantly improved numerical behavior at short scales [10]. The procedure was derived by considering a fluctuating LB model, making explicit use of the transformation between the populations $\left\{n_{i}\right\}$ and the modes [11].

The purpose of the present paper is to rederive the stochastic updating rule of Ref. [10] from a generalized latticegas model. The difference in our formulation lies in the introduction of an ensemble of population densities at each grid point, so that a fluctuating LB simulation is a single realization of this ensemble. There follows naturally a probability distribution, $P\left(\left\{n_{i}\right\}\right)$, for the set of populations $\left\{n_{i}\right\}$ at a position $\vec{r}$ and time $t$. The equilibrium distribution at a single site can be derived by maximizing $P$ subject to the constraints of fixed mass and momentum densities, $\rho$ and $\vec{j}$. This distribution agrees with the standard equilibrium distribution for LB models [Eq. (6)] up to terms of order $u^{2}$. A similar procedure has been followed in deriving $H$-theorems for LB models [12-14], but these papers were not concerned with fluctuations.

A coarse-graining of the microscopic collision operator leads to a Langevin description for the nonconserved degrees of freedom. However, these stochastic collisions may also be viewed as a Monte Carlo process [15], satisfying the principle of detailed balance governed by $P\left(\left\{n_{i}\right\}\right)$. The procedure of Refs. [7,9] can be shown to violate detailed balance, while the improved version of Ref. [10] satisfies it.

In summary, our goal is to reconnect the lattice Boltzmann equation with its lattice gas origins, and thus to establish a firm statistical mechanical foundation for stochastic LB simulations, in addition to the usual connection to fluctuating hydrodynamics $[7,10]$. We believe this provides a comparable theoretical framework to that already available for other stochastic simulation methods, such as dissipative particle dynamics [16] and stochastic rotation dynamics [17]. This formulation also offers the possibility for future modifications and generalizations, for example, to thermal flows [18], models with nonideal equations of state $[19,20]$, or multicomponent mixtures [21].

The paper is organized as follows. In Sec. II we describe the underlying lattice-gas model, derive the probability distribution $P\left(\left\{n_{i}\right\}\right)$, and show that the most probable value for $\left\{n_{i}\right\}$ is equivalent to Eq. (6). In Sec. III we consider small fluctuations around the equilibrium distribution. We show they are approximately Gaussian distributed, with the level of thermal fluctuations governed by the degree of coarse graining: a given amount of mass on a lattice site can be distributed between many particles, in which case the fluctuations are small, or between few, in which case they are large. In this way we can adjust the level of fluctuations while keeping the temperature fixed. In Sec. IV we construct a stochastic collision operator such that detailed balance is satisfied. From this, we derive the random stresses at an individual site. In Sec. V we apply the Chapman-Enskog procedure [9] to the algorithm in order to find the behavior on the hydrodynamic scale; the deterministic and stochastic terms are here treated on an equal basis [22]. We then find that, on the macroscopic scale, the procedure yields exactly the stress correlations given by Landau and Lifshitz [5]. Section VI discusses how to choose parameters for a coupled particle-fluid system. Section VII summarizes our conclusions.

\section{SINGLE-SITE PROBABILITY DISTRIBUTION}

Historically, the lattice-Boltzmann model $[3,23]$ was developed from earlier work on lattice-gas (LG) models $[24,25]$, in which each velocity direction was occupied by at most one particle. Here, we imagine a generalized lattice-gas model (GLG) where each velocity direction can be occupied by many particles. Each particle has the same mass, but different velocity directions may have different mean populations, even in a fluid at rest. The microscopic state of the system at any given site is specified by a set of integers $\left\{\nu_{i}\right\}$ giving the occupancies of each direction. Then the update of the GLG is analogous to the standard LG or LB models, but with $\nu_{i}$ an integer as opposed to a Boolean or real variable:

$$
\nu_{i}\left(\vec{r}+\vec{c}_{i} h, t+h\right)=\nu_{i}^{\star}(\vec{r}, t)=\nu_{i}(\vec{r}, t)+\widetilde{\Delta}_{i}\left\{\nu_{i}(\vec{r}, t)\right\},
$$

where $\widetilde{\Delta}_{i}$ operates on $\left\{\nu_{i}\right\}$ to compute the change in population $\nu_{i}^{\star}-\nu_{i}$. While collisions may be both deterministic and microscopically reversible, we shall assume only that the collision operator satisfies detailed balance.

Without considering the collision rules in detail, we construct an equilibrium distribution from the following thought experiment. Consider a velocity direction, $i$, at a particular site, $\vec{r}$. Particles are drawn randomly from a large reservoir and assigned to the site $\vec{r}$ with velocity $\vec{c}_{i}$; the number of particles in the reservoir is assumed to be much larger than the number of particles selected, $\nu_{i}(\vec{r})$. Under these circumstances $\nu_{i}(\vec{r})$ follows a Poisson distribution,

$$
P\left(\nu_{i}\right)=\frac{\bar{\nu}_{i}^{\nu_{i}}}{\nu_{i} !} e^{-\bar{\nu}_{i}},
$$

with a mean number of particles $\bar{\nu}_{i}$, and a variance

$$
\left\langle\nu_{i}^{2}\right\rangle-\left\langle\nu_{i}\right\rangle^{2}=\bar{\nu}_{i} .
$$

Let $m_{p}$ be the mass of a particle and $\mu=m_{p} / b^{d}$, where $b$ is the lattice spacing and $d$ is the spatial dimension. Then $n_{i}$ $=\mu \nu_{i}$, and

$$
\left\langle n_{i}^{2}\right\rangle-\left\langle n_{i}\right\rangle^{2}=\mu\left\langle n_{i}\right\rangle .
$$

The fluctuations in mass density at a site are controlled by the mass of an LB particle: small $m_{p}$ means that the mass is distributed onto many particles, and therefore fluctuations are small. For fixed $m_{p}, \mu$ (and therefore the level of fluctuations) becomes large as $b$ decreases. This is natural, since a fine spatial resolution means fewer particles per cell, and larger fluctuations relative to the mean. 
If we now imagine sampling each velocity with an independent reservoir, but taking only those sets of populations which produce specific values for the total mass and momentum, the probability density for the occupation numbers is (except for normalization)

$$
P\left(\left\{\nu_{i}\right\}\right) \propto\left(\prod_{i} \frac{\bar{\nu}_{i}^{\nu_{i}}}{\nu_{i} !} e^{-\bar{\nu}_{i}}\right) \delta\left(\mu \sum_{i} \nu_{i}-\rho\right) \delta\left(\mu \sum_{i} \nu_{i} \vec{c}_{i}-\vec{j}\right) .
$$

Using Stirling's approximation for $\nu_{i} \gg 1$, we can write the distribution in terms of the entropy associated with the occupation numbers,

$$
S\left(\left\{\nu_{i}\right\}\right)=-\sum_{i}\left(\nu_{i} \ln \nu_{i}-\nu_{i}-\nu_{i} \ln \bar{\nu}_{i}+\bar{\nu}_{i}\right),
$$

and the constraints:

$$
P\left(\left\{\nu_{i}\right\}\right) \propto \exp \left[S\left(\left\{\nu_{i}\right\}\right)\right] \delta\left(\mu \sum_{i} \nu_{i}-\rho\right) \delta\left(\mu \sum_{i} \nu_{i} \vec{c}_{i}-\vec{j}\right) .
$$

The equilibrium distribution, $\nu_{i}^{e q}$, can be found by maximizing $S$, treating $\nu_{i}$ as a continuous variable, and taking into account the mass and momentum constraints via Lagrange multipliers, $\lambda_{\rho}$ and $\overrightarrow{\lambda_{j}}$, respectively:

$$
\begin{gathered}
\frac{\partial S}{\partial \nu_{i}}+\lambda_{\rho}+\vec{\lambda}_{j} \cdot \vec{c}_{i}=0, \\
\mu \sum_{i} \nu_{i}-\rho=0, \\
\mu \sum_{i} \nu_{i} \vec{c}_{i}-\vec{j}=0 .
\end{gathered}
$$

It should be noted that this procedure is closely related to the determination of an entropy function for the LB equation [13]. Equation (15) can be solved to give the equilibrium populations in terms of the Lagrange multipliers,

$$
\nu_{i}^{e q}=\bar{\nu}_{i} \exp \left(\lambda_{\rho}+\vec{\lambda}_{\vec{j}} \cdot \vec{c}_{i}\right)
$$

which are then determined from the constraints, Eqs. (16) and (17), substituting $\nu_{i}^{e q}$ for $\nu_{i}$.

The mean populations in the absence of constraints, $\left\{\bar{\nu}_{i}\right\}$, can be expressed in terms of the mean number of particles at a site,

$$
\bar{\nu}_{i}=\bar{\nu} a^{c_{i}},
$$

where $\bar{\nu}=\Sigma_{i} \bar{\nu}_{i}$. The symmetry of the lattice constrains the weights, $a^{c_{i}}$, to be dependent on the speed of the particle, but not its direction. Thus for a lattice with cubic symmetry,

$$
\begin{gathered}
\sum_{i} a^{c_{i}}=1, \\
\sum_{i} a^{c_{i}} c_{i \alpha}=0,
\end{gathered}
$$

$$
\begin{gathered}
\sum_{i} a^{c_{i}} c_{i \alpha} c_{i \beta}=\sigma_{2} \delta_{\alpha \beta}, \\
\sum_{i} a^{c_{i}} c_{i \alpha} c_{i \beta} c_{i \gamma}=0,
\end{gathered}
$$

where $\delta_{\alpha \beta}$ is the Kronecker delta, and $\sigma_{2}$ is a constant with units $(b / h)^{2}$.

A solution of the nonlinear equations (16)-(18) requires an iterative numerical procedure, but it is more practical to seek an approximate expression for the equilibrium distribution in the limit that $\overrightarrow{\lambda_{j}} \cdot \vec{c}_{i}$ is small [14]. To second order in $\overrightarrow{\lambda_{j}}$, the mass and momentum constraints yield:

$$
\mu \bar{\nu} e^{\lambda} \rho\left(1+\frac{\sigma_{2} \lambda_{\vec{j}}^{2}}{2}\right)=\rho,
$$

$$
\mu \bar{\nu} e^{\lambda} \sigma_{2} \vec{\lambda} \vec{j}=\rho \vec{u}
$$

Inserting these results into Eq. (18), we find the equilibrium distribution can be written in the form of Eq. (6),

$$
n_{i}^{e q}=\rho a^{c_{i}}\left(1+\frac{\vec{u} \cdot \vec{c}_{i}}{\sigma_{2}}+\frac{\left(\vec{u} \cdot \vec{c}_{i}\right)^{2}}{2 \sigma_{2}^{2}}-\frac{u^{2}}{2 \sigma_{2}}\right) .
$$

For the sake of completeness, we now briefly mention the procedure $[4,9]$ to determine the weights $a^{c_{i}}$ such that the LB model is consistent with hydrodynamics. This requires that the second moment of the equilibrium distribution,

$$
\Pi_{\alpha \beta}^{e q}=\sum_{i} n_{i}^{e q} c_{i \alpha} c_{i \beta}
$$

should equal the Euler stress $p \delta_{\alpha \beta}+\rho u_{\alpha} u_{\beta}$, with the pressure given by the ideal gas equation of state, $p=\rho k_{B} T / m_{p}$, where $k_{B}$ is Boltzmann's constant and $T$ is the absolute temperature. For an isothermal gas of particles of mass $m_{p}, k_{B} T=m_{p} c_{\varepsilon}^{2}$, and therefore the equation of state is also given by $p=\rho c_{s}^{2}$, with $c_{s}$ the speed of sound.

To evaluate $\Pi_{\alpha \beta}^{e q}$ we require the fourth moment of $\left\{a^{c_{i}}\right\}$, which from cubic symmetry must be of the form

$$
\sum_{i} a^{c_{i}} c_{i \alpha} c_{i \beta} c_{i \gamma} c_{i \delta}=\kappa_{4} \delta_{\alpha \beta \gamma \delta}+\sigma_{4}\left(\delta_{\alpha \beta} \delta_{\gamma \delta}+\delta_{\alpha \gamma} \delta_{\beta \delta}+\delta_{\alpha \delta} \delta_{\beta \gamma}\right),
$$

where $\delta_{\alpha \beta \gamma \delta}$ is unity if all four indexes are the same and zero otherwise; $\kappa_{4}$ and $\sigma_{4}$ have units of $(b / h)^{4}$. Consistency between Eq. (27) and the Euler stress requires that

$$
\begin{gathered}
\sigma_{2}=c_{s}^{2}=k_{B} T / m_{p}, \\
\sigma_{4}=\sigma_{2}^{2}, \\
\kappa_{4}=0 .
\end{gathered}
$$

These conditions, together with the normalization condition, $\sum_{i} a^{c_{i}}=1$, determine the weights uniquely for a model with three different speeds. For example, for the D3Q19 model [4] (19 velocities on a three-dimensional simple cubic lattice), $a^{0}=1 / 3$ for the stationary particles, $a^{1}=1 / 18$ for the 
six nearest-neighbor directions, and $a^{\sqrt{2}}=1 / 36$ for the 12 next-nearest-neighbor directions: the sound speed is then $c_{s}^{2}$ $=(1 / 3)(b / h)^{2}$. In the D2Q9 model [4] (nine velocities on a two-dimensional square lattice) the weights are $a^{0}=4 / 9, a^{1}$ $=1 / 9$, and $a^{\sqrt{2}}=1 / 36$; the sound speed is again $c_{s}^{2}=(1 / 3)$ $\times(b / h)^{2}$.

\section{SINGLE-SITE FLUCTUATIONS}

We now consider the distribution of small fluctuations in the mass densities associated with each velocity direction, $n_{i}^{n e q}=n_{i}-n_{i}^{e q}$. Using the results of the Appendix to incorporate the constraints, and converting from fluctuations in $\nu_{i}$ to fluctuations in $n_{i}$,

$$
P\left(\left\{n_{i}^{n e q}\right\}\right) \propto \exp \left(-\sum_{i} \frac{\left(n_{i}^{n e q}\right)^{2}}{2 \mu n_{i}^{e q}}\right) \delta\left(\sum_{i} n_{i}^{n e q}\right) \delta\left(\sum_{i} \vec{c}_{i} n_{i}^{n e q}\right) .
$$

The variance in the fluctuations depends on direction, but, since $n_{i}^{\text {neq }}$ is already a small quantity in comparison with $n_{i}^{e q}$, we will approximate the variance by the low-velocity limit,

$$
\lim _{\vec{u} \rightarrow 0} n_{i}^{e q}=\rho a^{c_{i}} .
$$

The velocity dependence of the fluctuations in the GLG model is a consequence of the broken Galilean invariance, which is only entirely restored in the limit $u \rightarrow 0$. However, the approximation in Eq. (33) makes no difference to the macroscopic dynamics of the fluctuating LB model [Eqs. (82)-(85)], since the stress fluctuations are already secondorder in the Chapman-Enskog expansion.

We now introduce normalized fluctuations $x_{i}$, via the definition

$$
n_{i}^{n e q}=\sqrt{\mu \rho a^{c}} x_{i},
$$

and transform Eq. (32) to the simplified expression

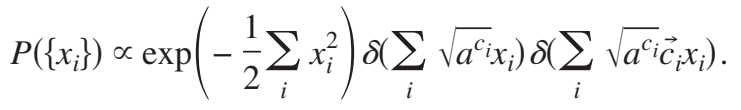

Equations (6), (34), and (35) define the statistics of our fluctuating LB model. Equation (35) is entirely consistent with the proposed GLG model, within the approximation expressed in Eq. (33).

The LB collision operator can be conveniently represented in terms of modes, which are linear combinations of the mass densities, $\left\{n_{i}\right\}$ [11], with basis vectors constructed from orthogonal polynomials in the velocity set $\left\{\vec{c}_{i}\right\}$. There is more than one possible choice for these basis vectors [26], and we use the "weighted" set $[10,26]$, for which only the hydrodynamic modes (mass density, momentum density, and stress) have a projection on the equilibrium distribution. We then write the nonequilibrium distribution as an orthonormal transformation of the scaled variables, $x_{i}$ :

$$
m_{k}=\sum_{i} \hat{e}_{k i} x_{i}
$$

$$
x_{i}=\sum_{k} \hat{e}_{k i} m_{k},
$$

where $m_{k}$ is the amplitude of the $k$ th mode, and the basis vectors satisfy the orthonormality conditions

$$
\sum_{i} \hat{e}_{k i} \hat{e}_{l i}=\delta_{k l} \text {. }
$$

It should be noted that the basis vectors $\hat{e}_{k i}$ are different from the $e_{k i}$ defined in Ref. [26], since there the transformation was for unscaled variables, $n_{i}$, rather than the scaled variables, $x_{i}$, used here. The essential physics of the transformation is, however, unchanged; the present expressions are just a reparametrization. The basis vectors $\hat{e}_{k i}$ are related to the weighted basis vectors used in Ref. [26]:

$$
\hat{e}_{k i}=\sqrt{\frac{a^{c_{i}}}{w_{k}}} e_{k i},
$$

where $w_{k}$ is the length of the $k$ th basis vector,

$$
w_{k}=\sum_{i} a^{c_{i}} e_{k i}^{2} \text {. }
$$

The hydrodynamic modes, mass density, momentum density, and stress, can be written in a model-independent form. Explicitly,

$$
\hat{e}_{0 i}=\sqrt{a^{c_{i}}}
$$

for the mass mode, and

$$
\hat{e}_{\alpha i}=\sqrt{\frac{a^{c_{i}}}{c_{s}^{2}}} c_{i \alpha}, \quad \alpha=1, \ldots, d
$$

for the momentum modes. Note that in our formalism $m_{0}$ and $m_{\alpha}(\alpha=1, \ldots, d)$ are zero.

In addition to the conserved modes, there are $d(d+1) / 2$ viscous modes: one bulk mode, $d-1$ shear modes involving diagonal elements of the $\vec{c}_{i} \vec{c}_{i}$ tensor, and $d(d-1) / 2$ offdiagonal elements. The bulk stress mode is given by

$$
\hat{e}_{d+1, i}=\frac{1}{c_{s}^{2}} \sqrt{\frac{a^{c_{i}}}{2 d}}\left(\vec{c}_{i}^{2}-d c_{s}^{2}\right),
$$

where orthogonality to the mass mode is assured by Schmidt orthogonalization. There is a shear mode of the form

$$
\hat{e}_{d+2, i}=\frac{1}{c_{s}^{2}} \sqrt{\frac{a^{c_{i}}}{2 d(d-1)}}\left(d c_{i x}^{2}-\vec{c}_{i}^{2}\right),
$$

and $d-2$ shear modes of the form $(d>2)$

$$
\hat{e}_{d+3, i}=\frac{\sqrt{a^{c_{i}}}}{2 c_{s}^{2}}\left(c_{i y}^{2}-c_{i z}^{2}\right),
$$

together with additional modes formed by cyclic permutations of the Cartesian indexes. The $d(d-1) / 2$ off-diagonal shear stresses are of the form

$$
\hat{e}_{2 d+1, i}=\frac{\sqrt{a^{c_{i}}}}{c_{s}^{2}} c_{i x} c_{i y}
$$

together with cyclic permutations. 
TABLE I. Basis vectors of the D2Q9 model. Each row corresponds to a different basis vector, with the actual polynomial in $\hat{c}_{i \alpha}$ shown in the second column; the components of $\hat{c}_{i \alpha}=c_{i \alpha} h / b$ are normalized to unity. The orthonormal basis vectors $\hat{e}_{k i}$ can be obtained from the table using Eq. (39), $\hat{e}_{k i}=\sqrt{a^{c_{i}} / w_{k}} e_{k i}$ : the normalizing factor for each basis vector is in the third column.

\begin{tabular}{ccc}
\hline \hline$k$ & $e_{k i}$ & $w_{k}$ \\
\hline 0 & 1 & 1 \\
1 & $\hat{c}_{i x}$ & $1 / 3$ \\
2 & $\hat{c}_{i y}$ & $1 / 3$ \\
3 & $3 \hat{c}_{i}^{2}-2$ & 4 \\
4 & $2 \hat{c}_{i x}^{2}-\hat{c}_{i}^{2}$ & $4 / 9$ \\
5 & $\hat{c}_{i x} \hat{c}_{i y}$ & $1 / 9$ \\
6 & $\left(3 \hat{c}_{i}^{2}-4\right) \hat{c}_{i x}$ & $2 / 3$ \\
7 & $\left(3 \hat{c}_{i}^{2}-4\right) \hat{c}_{i y}$ & $2 / 3$ \\
8 & $9 \hat{c}_{i}^{4}-15 \hat{c}_{i}^{2}+2$ & 16 \\
\hline \hline
\end{tabular}

All these vectors are mutually orthogonal. Further orthogonal vectors, whose span are the kinetic (or "ghost" [10]) modes, may be constructed in terms of higher-order polynomials of $\vec{c}_{i}$ [11]; these are model dependent. Complete sets of basis vectors [26] for the D2Q9 and D3Q19 LB models [4] are given in Tables I and II, respectively.

TABLE II. Basis vectors of the D3Q19 model. Each row corresponds to a different basis vector, with the actual polynomial in $\hat{c}_{i \alpha}$ shown in the second column; the components of $\hat{c}_{i \alpha}=c_{i \alpha} h / b$ are normalized to unity. The orthonormal basis vectors $\hat{e}_{k i}$ can be obtained from the table using Eq. (39), $\hat{e}_{k i}=\sqrt{a^{c_{i}} / w_{k}} e_{k i}$ : the normalizing factor for each basis vector is in the third column.

\begin{tabular}{ccc}
\hline \hline$k$ & $e_{k i}$ & $w_{k}$ \\
\hline 0 & 1 & 1 \\
1 & $\hat{c}_{i x}$ & $1 / 3$ \\
2 & $\hat{c}_{i y}$ & $1 / 3$ \\
3 & $\hat{c}_{i z}$ & $1 / 3$ \\
4 & $\hat{c}_{i}^{2}-1$ & $2 / 3$ \\
5 & $3 \hat{c}_{i x}^{2}-\hat{c}_{i}^{2}$ & $4 / 3$ \\
6 & $\hat{c}_{i y}^{2}-\hat{c}_{i z}^{2}$ & $4 / 9$ \\
7 & $\hat{c}_{i x} \hat{c}_{i y}$ & $1 / 9$ \\
8 & $\hat{c}_{i y} \hat{c}_{i z}$ & $1 / 9$ \\
9 & $\hat{c}_{i z} \hat{c}_{i x}$ & $1 / 9$ \\
10 & $\left(3 \hat{c}_{i}^{2}-5\right) \hat{c}_{i x}$ & $2 / 3$ \\
11 & $\left(3 \hat{c}_{i}^{2}-5\right) \hat{c}_{i y}$ & $2 / 3$ \\
12 & $\left(3 \hat{c}_{i}^{2}-5\right) \hat{c}_{i z}$ & $2 / 3$ \\
13 & $\left(\hat{c}_{i y}^{2}-\hat{c}_{i z}^{2} \hat{c}_{i x}\right.$ & $2 / 9$ \\
14 & $\left(\hat{c}_{i z}^{2}-\hat{c}_{i x}^{2}\right) \hat{c}_{i y}$ & $2 / 9$ \\
15 & $\left(\hat{c}_{i x}^{2}-\hat{c}_{i y}^{2}\right) \hat{c}_{i z}$ & $2 / 9$ \\
16 & $3 \hat{c}_{i}^{4}-6 \hat{c}_{i}^{2}+1$ & 2 \\
17 & $\left(2 \hat{c}_{i}^{2}-3\right)\left(3 \hat{c}_{i x}^{2}-\hat{c}_{i}^{2}\right)$ & $4 / 3$ \\
18 & $\left(2 \hat{c}_{i}^{2}-3\right)\left(\hat{c}_{i y}^{2}-\hat{c}_{i z}^{2}\right)$ & $4 / 9$ \\
\hline \hline
\end{tabular}

Equation (35) can be rewritten using Eqs. (37) and (38) to give the nonequilibrium probability distribution of the modes $m_{k}$,

$$
P\left(\left\{m_{k}\right\}\right) \propto \exp \left(-\frac{1}{2} \sum_{k} m_{k}^{2}\right) \prod_{i \leq d} \delta\left(m_{i}\right) \propto \exp \left(-\frac{1}{2} \sum_{k>d} m_{k}^{2}\right) .
$$

There is no contribution to $P$ from the conserved modes.

\section{STOCHASTIC COLLISIONS AS A MONTE CARLO PROCESS}

In this section we construct a stochastic collision operator, viewed as a Monte Carlo process, and consider the local dynamics at the level of a single lattice site. In the next section (Sec. V) we will consider the global dynamics, through a Chapman-Enskog expansion. A deterministic collision operator at the microscopic level is quite complicated to construct, even for the simplest three-dimensional LG models [27], and cannot be easily extended to the larger number of particles in Eq. (8). Collision rules are much easier to construct at the Boltzmann level [3]; the stochastic update from precollision to postcollision populations, $n_{i}$ $\rightarrow n_{i}^{\star}$, is facilitated by making the transition between modes, $m_{k} \rightarrow m_{k}^{\star}$, since each degree of freedom is then independent. Denoting a transition probability between the pre- and postcollision states of a particular mode $m$ by $\omega\left(m \rightarrow m^{\star}\right)$, the condition of detailed balance, governed by the distribution in Eq. (47), reads

$$
\frac{\omega\left(m \rightarrow m^{\star}\right)}{\omega\left(m^{\star} \rightarrow m\right)}=\frac{\exp \left(-m^{\star 2} / 2\right)}{\exp \left(-m^{2} / 2\right)} .
$$

A simulation at the hydrodynamic level does not need to satisfy this condition, and typically does not, but it is essential for a proper thermal equilibrium of the LB fluid.

There are many possible realizations of Eq. (48): one well-known example is the Metropolis method, involving a trial move followed by a stochastic acceptance or rejection step to enforce detailed balance. Here we consider the linear relaxation model typically used in LB simulations, balanced by Gaussian noise:

$$
m^{\star}=\gamma m+\varphi r
$$

where $\gamma$ is related to an eigenvalue of the linearized collision operator, $\gamma=1+\lambda$ (see Eq. (8) of Ref. [26]), and $r$ is a Gaussian random variable with zero mean and unit variance. The dissipation parameter $\gamma$ is restricted by the linear stability limit, $|\gamma| \leq 1$, with the case $\gamma<0$ corresponding to "overrelaxation". Equation (49) has the technical advantage of being rejection-free, and the conceptual advantage of enabling an analytic calculation to be made at the Chapman-Enskog level (see Sec. V).

The parameter $\varphi$ must be adjusted to satisfy detailed balance, Eq. (48), using the relation [Eq. (49)] $r=\varphi^{-1}\left(m^{\star}\right.$ $-\gamma m)$. Since the transition probability for $m \rightarrow m^{\star}$ is identical to the probability for generating the value of $r$ that gives $m^{\star}$ from $m$, 


$$
\omega\left(m \rightarrow m^{\star}\right)=\left(2 \pi \varphi^{2}\right)^{-1 / 2} \exp \left[-\left(m^{\star}-\gamma m\right)^{2} / 2 \varphi^{2}\right] .
$$

There is a similar expression for the reverse transition, $m^{\star}$ $\rightarrow m$, with $m^{\star}$ and $m$ interchanged. From Eq. (48), we then find that detailed balance is satisfied for

$$
\varphi=\left(1-\gamma^{2}\right)^{1 / 2} .
$$

Thus the case $\gamma=1$ corresponds to a conserved mode, while $\gamma=0$ corresponds to $m^{\star}$ being entirely random, with no memory of its previous value.

Each mode, $m_{k}$, in the LB model is assigned its own relaxation rate $\gamma_{k}$, subject to the constraints of symmetry and conservation laws; the conserved modes $(k \leq d)$ require that $\gamma_{k}=1$. For the bulk stress we choose a value $\gamma_{b}$, and for the $(d+2)(d-1) / 2$ shear stresses a single value $\gamma_{s}$. In Refs. $[7,9,10]$ the kinetic modes were updated with $\gamma_{k}=0$, but it is possible to achieve more accurate boundary conditions with a proper tuning of the kinetic eigenvalues $[26,28]$. Equation (51) ensures that detailed balance is satisfied for all choices of $\gamma_{k}$. A purely deterministic LB model is obtained by setting $\varphi_{k}=0$ for all modes; physically, this corresponds to the limit of $m_{p} \rightarrow 0$, or $\bar{\nu}_{i} \rightarrow \infty$.

The original derivation of the fluctuating LB model $[7,9]$ is obtained by setting $\gamma_{k}=\varphi_{k}=0$ for all the kinetic modes, but choosing the variance of the stresses according to Eq. (51). The kinetic modes are projected out at every time step by this collision rule, and $\omega\left(m_{k} \rightarrow m_{k}^{\star}=0\right)=1$. However, there is no route back to the precollisional state, $\omega\left(m_{k}^{\star}=0 \rightarrow m_{k}\right)=0$, and detailed balance [Eq. (48)] is clearly violated. Nevertheless, this model still yields the correct fluctuating hydrodynamics in the limit of large length scales [9], as is shown by the analysis in Sec. V. Treating all the nonconserved modes on an equal basis [10] satisfies detailed balance on all scales, and is entirely equivalent to Eqs. (48)-(51).

As a general rule, proper thermalization requires as many random variables as there are degrees of freedom in the system (not counting the conserved variables). However, in the special case where $\gamma_{k}=0$ for all kinetic modes, the deterministic LB model can be propagated forward in time from the mass, momentum, and stress at each lattice site. Thus it is tempting to conclude that in this instance only the stress modes should be thermalized [6-8], since the $\left\{n_{i}\right\}$ play the role of auxiliary variables. However, this is incorrect; if both $\gamma_{k}=0$ and $\varphi_{k}=0$ for any mode, it is impossible to reconstruct the reverse trajectory and the system will not reach thermal equilibrium. The number of degrees of freedom is only welldefined, therefore, when all $\gamma_{k} \neq 0$. Situations where some $\gamma_{k}=0$ should be treated as a limit of finite $\gamma_{k}$, and continuity tells us that the number of random variables should be the same.

The update rule in Eq. (49), with $\gamma>0$, is an exact solution of a continuous Langevin equation $[29,30]$,

$$
\frac{d}{d t} m=-\Gamma m+\xi
$$

with $\langle\xi(t)\rangle=0$ and $\left\langle\xi(t) \xi\left(t^{\prime}\right)\right\rangle=2 \Gamma \delta\left(t-t^{\prime}\right)$. Integrating Eq. (52) from $t=0$ to $t=h$ (i.e., one LB time step) gives Eq. (49), with $\gamma=\exp (-\Gamma h)$. The standard first-order Euler approxima- tion to Eq. (52) corresponds to $\gamma=1-\Gamma h$, and is only valid for small $\Gamma h$. By contrast Eq. (49) does not impose any restriction on the time step.

For the Chapman-Enskog analysis in Sec. V, we will need the collisional update of the nonequilibrium stress tensor, $\Pi_{\alpha \beta}^{n e q}=\Sigma_{i} n_{i}^{n e q} c_{i \alpha} c_{i \beta}$; the equilibrium part of the stress is unchanged by the collision process. We first decompose $\Pi_{\alpha \beta}^{\text {neq }}$ into a multiple of the unit tensor (bulk stress), and a traceless part (shear stresses), denoted by an overbar:

$$
\Pi_{\alpha \beta}^{n e q}=\bar{\Pi}_{\alpha \beta}^{n e q}+\frac{1}{d} \Pi_{\gamma \gamma}^{n e q} \delta_{\alpha \beta},
$$

where we have used the Einstein summation convention for the Cartesian components. The change in the nonequilibrium stress tensor at a lattice site, due to collisions, can be determined from Eqs. (49) and (51),

$$
\begin{aligned}
& \bar{\Pi}_{\alpha \beta}^{\star n e q}=\gamma_{s} \bar{\Pi}_{\alpha \beta}^{n e q}+\bar{R}_{\alpha \beta}, \\
& \Pi_{\alpha \alpha}^{\star n e q}=\gamma_{b} \Pi_{\alpha \alpha}^{n e q}+R_{\alpha \alpha} .
\end{aligned}
$$

The variables $R_{\alpha \beta}$ are Gaussian random variables with zero mean; in addition $\bar{R}_{\alpha \beta}$ is traceless. The covariance matrix $\left\langle R_{\alpha \beta} R_{\gamma \delta}\right\rangle$ is determined by the variances of the stochastic stress modes. The calculation can be simplified by observing that the matrix is a fourth rank tensor and is therefore isotropic by the symmetries of the LB model,

$$
\left\langle R_{\alpha \beta} R_{\gamma \delta}\right\rangle=R_{1}\left(\delta_{\alpha \gamma} \delta_{\beta \delta}+\delta_{\alpha \delta} \delta_{\beta \gamma}\right)+R_{2} \delta_{\alpha \beta} \delta_{\gamma \delta} .
$$

The unknown constants, $R_{1}$ and $R_{2}$, can be determined from special cases. For example, in the D3Q19 model defined in Table II,

$$
\Pi_{x y}^{n e q}=\sqrt{\mu \rho} c_{s}^{2} m_{7},
$$

and therefore, from Eqs. (49) and (54),

$$
\left\langle R_{x y}^{2}\right\rangle=\mu \rho c_{s}^{4}\left(1-\gamma_{s}^{2}\right)=R_{1},
$$

where the final equality follows from Eq. (56). Similarly,

$$
\Pi_{y y}^{n e q}-\Pi_{z z}^{n e q}=2 \sqrt{\mu \rho} c_{s}^{2} m_{6},
$$

and therefore

$$
\left\langle\left(R_{y y}-R_{z z}\right)^{2}\right\rangle=4 \mu \rho c_{s}^{4}\left(1-\gamma_{s}^{2}\right)=4 R_{1} .
$$

This result is consistent with Eq. (56), which demonstrates that the fluctuating stresses are indeed isotropic. Finally, the fluctuations in the trace, $R_{\alpha \alpha}$, are related to $\gamma_{b}$ :

$$
\left\langle R_{\alpha \alpha} R_{\beta \beta}\right\rangle=6 \mu \rho c_{s}^{4}\left(1-\gamma_{b}^{2}\right)=6 R_{1}+9 R_{2} .
$$

The general expression for the covariance in the random stresses is

$$
\frac{\left\langle R_{\alpha \beta} R_{\gamma \delta}\right\rangle}{\mu \rho c_{s}^{4}}=\left(1-\gamma_{s}^{2}\right)\left(\delta_{\alpha \gamma} \delta_{\beta \delta}+\delta_{\alpha \delta} \delta_{\beta \gamma}\right)+\frac{2}{d}\left(\gamma_{s}^{2}-\gamma_{b}^{2}\right) \delta_{\alpha \beta} \delta_{\gamma \delta} .
$$

This covariance matrix is different from the global fluctuations in stress, which are superposed onto the hydrodynamic modes (Sec. V). 


\section{CHAPMAN-ENSKOG EXPANSION}

In order to determine the behavior on hydrodynamic length and time scales, we apply the Chapman-Enskog method to the stochastic dynamics of the fluctuating LB model. We modify the derivation of Ref. [9] to include thermal fluctuations: for an alternative procedure, see Ref. [31]. Here, the expansion parameter $\varepsilon$ is used to separate the lattice scale, $\vec{r}$, from the hydrodynamic scale, $\vec{r}_{1}=\varepsilon \vec{r}$. Thus $\partial_{\alpha}$ $=\varepsilon \partial_{\alpha}^{1}$, with the notation $\partial_{\alpha}=\partial / \partial r_{\alpha}$.

Since the collision operator is local in space and time, the nonequilibrium distribution is also taken to be of order $\varepsilon$ : $n_{i}^{n e q}=\varepsilon n_{i}^{1}$, with $n_{i}^{1}$ of order unity in the $\varepsilon$ expansion,

$$
n_{i}=n_{i}^{e q}+\varepsilon n_{i}^{1}
$$

We use the usual multiple time scale expansion [32], $\partial_{t}$ $=\varepsilon \partial_{t_{1}}+\varepsilon^{2} \partial_{t_{2}}$, to separate the convective $\left(t_{1}\right)$ and diffusive $\left(t_{2}\right)$ relaxation processes. The left-hand side of Eq. (1) is expanded about $(\vec{r}, t)$ in a Taylor series with respect to $h$ : to first order in $\varepsilon$,

$$
\left(\partial_{t_{1}}+c_{i \alpha} \partial_{\alpha}^{1}\right) n_{i}^{e q}=h^{-1} \Delta_{i}
$$

Multiplying this equation by one of the basis vectors and summing over all the directions, we obtain the equations for the dynamics of the fluctuating LB model on the $t_{1}$ time scale,

$$
\partial_{t_{1}} \sum_{i} n_{i}^{e q} e_{k i}+\partial_{\alpha}^{1} \sum_{i} n_{i}^{e q} c_{i \alpha} e_{k i}=h^{-1} \sum_{i} \Delta_{i} e_{k i} .
$$

Note that we use the $e_{k i}$ basis vectors here, in conjunction with $\left\{n_{i}^{e q}\right\}$ and $\left\{n_{i}^{n e q}\right\}$, not the normalized basis vectors $\hat{e}_{k i}$, which are for the $\left\{x_{i}\right\}$.

When applied to the conserved degrees of freedom, $k$ $\leq d$, Eq. (65) leads to the inviscid fluid equations:

$$
\begin{gathered}
\partial_{t_{1}} \rho+\partial_{\alpha}^{1} j_{\alpha}=0, \\
\partial_{t_{1}} j_{\alpha}+\partial_{\beta}^{1}\left(\rho c_{s}^{2} \delta_{\alpha \beta}+\rho u_{\alpha} u_{\beta}\right)=0 .
\end{gathered}
$$

Similarly, for the stress modes, $d<k \leq\left(d^{2}+3 d\right) / 2$, we find:

$$
\begin{aligned}
& \partial_{t_{1}}\left(\rho c_{s}^{2} \delta_{\alpha \beta}+\rho u_{\alpha} u_{\beta}\right)+c_{s}^{2}\left(\partial_{\alpha}^{1} j_{\beta}+\partial_{\beta}^{1} j_{\alpha}+\partial_{\gamma}^{1} j_{\gamma} \delta_{\alpha \beta}\right) \\
& \quad=h^{-1}\left(\Pi_{\alpha \beta}^{\star}-\Pi_{\alpha \beta}\right) .
\end{aligned}
$$

Evaluating the time derivatives in Eq. (68) gives a simplified expression for the nonequilibrium stress, apart from small terms of order $u^{3}$ [9],

$$
\Pi_{\alpha \beta}^{\star}-\Pi_{\alpha \beta}=h \rho c_{s}^{2}\left(\partial_{\alpha}^{1} u_{\beta}+\partial_{\beta}^{1} u_{\alpha}\right) .
$$

Thus, on the $t_{1}$ time scale, the viscous stresses fluctuate around a mean value that is slaved to the velocity gradient, $\partial_{\alpha}^{1} u_{\beta}+\partial_{\beta}^{1} u_{\alpha}$.

The kinetic modes fluctuate around zero on the $t_{1}$ time scale, with at most a small correction of order $u^{2}$ :

$$
m_{k}^{\star}-m_{k}=O\left(u^{2}\right) .
$$

The equilibrium distribution contains polynomials in $\vec{c}_{i}$ up to second order, and is thus automatically orthogonal to the kinetic modes, which are made up of third-order and fourth- order polynomials in $\vec{c}_{i}$. Since the equilibrium distribution has no projection on the kinetic modes, the time-derivative in Eq. (65) vanishes identically for $k>\left(d^{2}+3 d\right) / 2$. However, the gradient term in Eq. (65) includes an additional factor of $\vec{c}_{i}$ : thus third-order polynomials survive, making small equilibrium contributions of order $u^{2}$ to the dynamics.

At order $\varepsilon^{2}$, the Boltzmann equation is

$$
\begin{gathered}
\partial_{t_{2}} n_{i}^{e q}+\partial_{t_{1}} n_{i}^{n e q}+c_{i \alpha} \partial_{\alpha}^{1} n_{i}^{n e q}+\frac{h}{2} \partial_{t_{1}}\left(\partial_{t_{1}}+c_{i \alpha} \partial_{\alpha}^{1}\right) n_{i}^{e q} \\
+\frac{h}{2} \partial_{\beta}^{1}\left(\partial_{t_{1}}+c_{i \alpha} \partial_{\alpha}^{1}\right) n_{i}^{e q} c_{i \beta}=0,
\end{gathered}
$$

where the terms have been grouped to suggest the most expedient means of calculation. Since only the hydrodynamic modes survive to the $t_{2}$ time scale, we consider just the modes up to $k=d$. It follows immediately from Eq. (71) and the conservations laws [Eqs. (66) and (67)] that the fluid is incompressible on the $t_{2}$ time scale,

$$
\partial_{t_{2}} \rho=0
$$

The momentum equation can be written as

$$
\partial_{t_{2}} j_{\alpha}+\partial_{\beta}^{1}\left\{\Pi_{\alpha \beta}^{n e q}+\frac{1}{2}\left(\Pi_{\alpha \beta}^{\star}-\Pi_{\alpha \beta}\right)\right\}=0,
$$

where we can use Eq. (69) to substitute the velocity gradients for $\Pi_{\alpha \beta}^{\star}-\Pi_{\alpha \beta}$. This is the usual lattice correction to the viscous momentum flux [9]. The kinetic modes make no contribution to the hydrodynamic variables, $\rho$ and $\vec{j}$, at long times.

The nonequilibrium stress can be calculated by combining the stress update rule, Eqs. (54) and (55), with Eq. (69). For example, from Eq. (54),

$$
\Pi_{x y}^{\star}-\Pi_{x y}^{e q}=\gamma_{s}\left(\Pi_{x y}-\Pi_{x y}^{e q}\right)+R_{x y}
$$

and from Eq. (69)

$$
\Pi_{x y}^{\star}-\Pi_{x y}=h \rho c_{s}^{2}\left(\partial_{x}^{1} u_{y}+\partial_{y}^{1} u_{x}\right) .
$$

Eliminating $\Pi_{x y}^{\star}$ from these two equations,

$$
\left(1-\gamma_{s}\right) \Pi_{x y}^{n e q}+h \rho c_{s}^{2}\left(\partial_{x}^{1} u_{y}+\partial_{y}^{1} u_{x}\right)=R_{x y} .
$$

In the general case, we again decompose the stress into its trace and traceless parts,

$$
\begin{aligned}
\Pi_{\alpha \beta}^{n e q}= & -\frac{h \rho c_{s}^{2}}{1-\gamma_{s}}\left(\partial_{\alpha}^{1} u_{\beta}+\partial_{\beta}^{1} u_{\alpha}-\frac{2}{d} \partial_{\gamma}^{1} u_{\gamma} \delta_{\alpha \beta}\right) \\
& -\frac{h \rho c_{s}^{2}}{1-\gamma_{b}}\left(\frac{2}{d} \partial_{\gamma}^{1} u_{\gamma} \delta_{\alpha \beta}\right)-Q_{\alpha \beta},
\end{aligned}
$$

where the random stress tensor on the macroscopic level is

$$
Q_{\alpha \beta}=-\frac{1}{1-\gamma_{s}} \bar{R}_{\alpha \beta}-\frac{1}{1-\gamma_{b}} \frac{1}{d} \delta_{\alpha \beta} R_{\gamma \gamma} .
$$

Equation (73) can now be rewritten in terms of the viscous and fluctuating stresses 


$$
\partial_{t_{2}} j_{\alpha}=\partial_{\beta}^{1} Q_{\alpha \beta}+\partial_{\beta}^{1}\left[\eta\left(\partial_{\alpha}^{1} u_{\beta}+\partial_{\beta}^{1} u_{\alpha}\right)+\left(\zeta-\frac{2 \eta}{d}\right) \partial_{\gamma}^{1} u_{\gamma} \delta_{\alpha \beta}\right] .
$$

The deterministic part of the stress tensor has the desired Newtonian form [5], with the usual expressions [9] for the shear viscosity $\eta$ and bulk viscosity $\zeta$ :

$$
\begin{gathered}
\eta=\frac{h \rho c_{s}^{2}}{2} \frac{1+\gamma_{s}}{1-\gamma_{s}}, \\
\zeta=\frac{h \rho c_{s}^{2}}{d} \frac{1+\gamma_{b}}{1-\gamma_{b}} .
\end{gathered}
$$

Combining the momentum transport on the $t_{1}$ and $t_{2}$ time scales we obtain the equations of fluctuating hydrodynamics [5],

$$
\begin{gathered}
\partial_{t} \rho+\partial_{\alpha}\left(\rho u_{\alpha}\right)=0, \\
\partial_{t}\left(\rho u_{\alpha}\right)+\partial_{\beta}\left(\rho u_{\alpha} u_{\beta}\right)+c_{s}^{2} \partial_{\alpha} \rho \\
=\partial_{\beta} Q_{\alpha \beta}+\partial_{\beta}\left[\eta\left(\partial_{\alpha} u_{\beta}+\partial_{\beta} u_{\alpha}\right)+\left(\zeta-\frac{2 \eta}{d}\right) \partial_{\gamma} u_{\gamma} \delta_{\alpha \beta}\right],
\end{gathered}
$$

with random stresses $Q_{\alpha \beta}$. These are Gaussian variables with zero mean and a covariance matrix that can be calculated from the analogous result on the microscopic level, Eq. (62):

$$
\left\langle Q_{\alpha \beta} Q_{\gamma \delta}\right\rangle=\frac{2 m_{p} c_{s}^{2}}{b^{d} h}\left[\eta\left(\delta_{\alpha \gamma} \delta_{\beta \delta}+\delta_{\alpha \delta} \delta_{\beta \gamma}\right)+\left(\zeta-\frac{2 \eta}{d}\right) \delta_{\alpha \beta} \delta_{\gamma \delta}\right] .
$$

This is the discrete analog of the covariance matrix of the fluctuating stresses given by Landau and Lifshitz [5]. The delta functions in space and time that appear in the continuum theory are here converted into factors $b^{-d}$ and $h^{-1}$. Thus the stress fluctuations depend on the discretization of space and time. Equation (84) can be made consistent with the amplitude of fluctuating stresses in Ref. [5] by choosing

$$
k_{B} T=m_{p} c_{s}^{2} .
$$

This is exactly the relation expected from the equation of state of an isothermal, ideal gas. In other words, our results are simultaneously consistent with macroscopic thermodynamics and fluctuating hydrodynamics.

\section{CHOICE OF PARAMETERS}

The fluctuating LB model has been used to simulate a range of soft-matter physics, such as colloidal suspensions [6] and polymer solutions [33,34]. In such cases it is necessary to match the LB parameters to the mass density, temperature, and viscosity of the molecular system. In addition there are two parameters that control the accuracy of the LB simulation without affecting the physics being simulated; namely the grid spacing, $b$, and the time step, $h$. The grid spacing must be related to the characteristic length scale of the physical system. For example, in coupling the LB fluid to soft matter, like polymer chains, colloidal particles, or membranes, the length would be the size of the object. For flow in complex geometries, it would be the channel width, while for simulations of turbulent flow, it would be the Kolmogorov length. This length scale, plus the desired spatial resolution, fixes the lattice spacing $b$ in absolute units. Choosing a suitable time step then automatically sets the speed of sound $c_{s}=\hat{c}_{s} b / h$, where $\hat{c}_{s}$ is a dimensionless property of the LB model; for example, in the D2Q9 and D3Q19 models $\hat{c}_{s}$ $=\sqrt{1 / 3}$. Typically, the sound speed will be unrealistically small for a dense liquid; however, this is not crucial since the LB method only runs in flow regimes where density fluctuations are negligible.

Once the length and time scales have been set, we can match the shear and bulk viscosities to the molecular system. Equations (80) and (81) suggest using $b$ and $h$ to compute nondimensional viscosities from the reference values,

$$
\begin{aligned}
& \hat{\eta}=\frac{\eta}{h \rho c_{s}^{2}}=\frac{\eta h}{\rho b^{2} \hat{c}_{s}^{2}}, \\
& \hat{\zeta}=\frac{\zeta}{h \rho c_{s}^{2}}=\frac{\zeta h}{\rho b^{2} \hat{c}_{s}^{2}} .
\end{aligned}
$$

The parameters $\gamma_{s}$ and $\gamma_{b}$ are then set by $\hat{\eta}$ and $\hat{\zeta}$ :

$$
\begin{aligned}
& \gamma_{s}=\frac{2 \hat{\eta}-1}{2 \hat{\eta}+1}, \\
& \gamma_{b}=\frac{d \hat{\zeta}-1}{d \hat{\zeta}+1} .
\end{aligned}
$$

Small time steps therefore imply that the LB simulation is run in the over-relaxation regime. The relaxation rates of the kinetic modes can be chosen for convenience $\left(\gamma_{k}=0\right)$ or to improve the accuracy of the boundary conditions $[26,28]$.

The remaining LB parameter is the particle mass, $m_{p}$, which must be fixed, for a given $b$ and $h$, so that the fluctuations in the LB fluid are consistent with the temperature, Eq. (85). The parameter $\mu=m_{p} / b^{d}$ determines the variance in the fluctuations [Eq. (32)],

$$
\mu=\frac{k_{B} T h^{2}}{\hat{c}_{s}^{2} b^{d+2}},
$$

from which we see that too fine a grid or too large a time step will cause an unacceptably high noise level. A stable simulation will require that the time step scales as $h \propto b^{d / 2+1}$ or $b^{5 / 2}$ in three dimensions, which is slightly more stringent than the usual diffusive scaling, $h \propto b^{2}$.

\section{CONCLUSIONS}

For models of the D3Q19 type, our analysis has shown that a fluctuating LB equation can be developed from statistical mechanical considerations. We have shown that the fluctuations are governed by the degree of coarse graining, and that the relevant parameter is the mass of the LB particle, 
$m_{p}$, which, for a given temperature, is determined by the discretization of space, $b$, and time, $h$. The temperature appearing in the equation of state is identical to that which controls the fluctuations, as it should be.

The beauty of the present approach is that one only needs to take care that the statistical properties are correct at the LB level. The correct fluctuation-dissipation theorem at the Navier-Stokes level is then an automatic consequence of the microscopic physics. We have introduced the principle of detailed balance into the LB model, which is the microscopic counterpart of the fluctuation-dissipation theorem used in previous work $[7,9,10]$. We have demonstrated that all nonconserved modes must be thermalized [10] in order to satisfy detailed balance; early implementations of the fluctuating LB model [7,9] did not preserve detailed balance. On the other hand, all these methods have been shown to be correct in the hydrodynamic limit. Only the stress fluctuations survive to long times, and fluctuations in the kinetic modes become asymptotically irrelevant. Nevertheless, practical simulations rarely probe the asymptotic limit, and then a procedure which is statistically correct on all length scales is clearly preferable.

\section{ACKNOWLEDGMENTS}

We thank R. Adhikari, M. E. Cates, and A. J. Wagner for very stimulating discussions on the subject. U.D.S. thanks the Volkswagen Foundation for support within the framework of the program "New conceptual approaches to modeling and simulation of complex systems." A.J.C.L. thanks the Alexander von Humboldt Foundation for supporting his stay at the Max Planck Institute for Polymer Research.

\section{APPENDIX: CONSTRAINED DISTRIBUTIONS}

Let us consider a constrained probability distribution $P\left(\left\{\nu_{i}\right\}\right)$ of the following general form:

$$
P\left(\left\{\nu_{i}\right\}\right) \propto \exp \left[S\left(\left\{\nu_{i}\right\}\right)\right] \prod_{j} \delta\left(\sum_{i} \nu_{i} \alpha_{i j}-q_{j}\right),
$$

where $S$ is a function of $\left\{\nu_{i}\right\}$, and $\alpha_{i j}$ and $q_{j}$ are constants. The constraints can be eliminated by making use of the Fourier representation of the delta function, $\delta(x)$ $=(2 \pi)^{-1} \int \exp (i k x) d k$ :

$$
P\left(\left\{\nu_{i}\right\}\right) \propto\left(\prod_{j} \int d k_{j}\right) \exp \left[\hat{S}\left(\left\{\nu_{i}\right\},\left\{k_{j}\right\}\right)\right],
$$

where

$$
\hat{S}\left(\left\{\nu_{i}\right\},\left\{k_{j}\right\}\right)=S\left(\left\{\nu_{i}\right\}\right)+i \sum_{j} k_{j}\left(\sum_{i} \nu_{i} \alpha_{i j}-q_{j}\right) .
$$

Now, let $\nu_{i}^{(0)}, k_{j}^{(0)}$ denote the saddle point of $\hat{S}$, which can be found by solving the system of equations:

$$
\begin{gathered}
\frac{\partial \hat{S}}{\partial \nu_{i}}=0 \Leftrightarrow \frac{\partial S}{\partial \nu_{i}}+i \sum_{j} k_{j} \alpha_{i j}=0, \\
\frac{\partial \hat{S}}{\partial k_{j}}=0 \Leftrightarrow \sum_{i} \nu_{i} \alpha_{i j}-q_{j}=0 .
\end{gathered}
$$

The solution $\nu_{i}^{(0)}$ satisfies the constraints in Eq. (A1) and is identical to the one obtained by maximizing $S$, taking into account the constraints via Lagrange multipliers, $i k_{j}$.

The second-order Taylor expansion of $\hat{S}$ around the saddle point is

$$
\hat{S}\left(\left\{\nu_{i}\right\},\left\{k_{j}\right\}\right)=\hat{S}\left(\left\{\nu_{i}^{(0)}\right\},\left\{k_{j}^{(0)}\right\}\right)+\sum_{i l} \beta_{i l} \delta \nu_{i} \delta \nu_{l}+i \sum_{i j} \alpha_{i j} \delta \nu_{i} \delta k_{j},
$$

where we have introduced the abbreviations

$$
\begin{gathered}
\beta_{i l}=\left.\frac{1}{2} \frac{\partial^{2} S}{\partial \nu_{i} \partial \nu_{l}}\right|_{\left\{\nu_{i}^{(0)}\right\}}, \\
\delta \nu_{i}=\nu_{i}-\nu_{i}^{(0)}, \\
\delta k_{j}=k_{j}-k_{j}^{(0)} .
\end{gathered}
$$

The probability distribution for $\delta \nu_{i}$ is then approximately Gaussian.

The expansion of $\hat{S}$ is now inserted into Eq. (A2). Ignoring the constant term, which can be absorbed in the normalization of $P$, and transforming to the new variables $\delta k_{j}$, we find

$$
\begin{aligned}
P\left(\left\{\delta \nu_{i}\right\}\right) \propto & {\left[\prod_{j} \int d\left(\delta k_{j}\right) \exp \left(i \delta k_{j} \sum_{i} \alpha_{i j} \delta \nu_{i}\right)\right] } \\
& \times \exp \left(\sum_{i l} \beta_{i l} \delta \nu_{i} \delta \nu_{l}\right) .
\end{aligned}
$$

Reintroducing delta functions, we obtain the final result

$$
P\left(\left\{\delta \nu_{i}\right\}\right) \propto \exp \left(\sum_{i l} \beta_{i l} \delta \nu_{i} \delta \nu_{l}\right) \prod_{j} \delta\left(\sum_{i} \alpha_{i j} \delta \nu_{i}\right) .
$$

Assuming the coefficients $\beta_{i j}$ form a negative-definite matrix (otherwise the Gaussian approximation would not make sense), the saddle point is a maximum in $P$.
[1] S. Succi, The Lattice Boltzmann Equation for Fluid Dynamics and Beyond (Oxford University Press, New York, 2001).

[2] R. Benzi, S. Succi, and M. Vergassola, Phys. Rep. 222, 145 (1992)
[3] F. Higuera, S. Succi, and R. Benzi, Europhys. Lett. 9, 345 (1989).

[4] Y. H. Qian, D. d'Humières, and P. Lallemand, Europhys. Lett. 17, 479 (1992). 
[5] L. D. Landau and E. M. Lifshitz, Fluid Mechanics (AddisonWesley, London, 1959).

[6] A. J. C. Ladd, Phys. Rev. Lett. 70, 1339 (1993).

[7] A. J. C. Ladd, J. Fluid Mech. 271, 285 (1994).

[8] A. J. C. Ladd, J. Fluid Mech. 271, 311 (1994).

[9] A. J. C. Ladd and R. Verberg, J. Stat. Phys. 104, 1191 (2001).

[10] R. Adhikari, K. Stratford, M. E. Cates, and A. J. Wagner, Europhys. Lett. 71, 473 (2005).

[11] D. D'Humières, I. Ginzburg, M. Kraftzyck, P. Lallemand, and L. S. Luo, Philos. Trans. R. Soc. London, Ser. A 360, 437 (2002).

[12] A. J. Wagner, Europhys. Lett. 44, 144 (1998).

[13] I. V. Karlin, A. Ferrante, and H. C. Öttinger, Europhys. Lett. 47, 182 (1999).

[14] B. M. Boghosian, P. J. Love, P. V. Coveney, I. V. Karlin, S. Succi, and J. Yepez, Phys. Rev. E 68, 025103(R) (2003).

[15] D. P. Landau and K. Binder, A Guide to Monte Carlo Simulations in Statistical Physics (Cambridge University Press, Cambridge, England, 2000).

[16] P. Español and P. Warren, Europhys. Lett. 30, 191 (1995).

[17] A. Malevanets and R. Kapral, J. Chem. Phys. 110, 8605 (1999).

[18] G. R. McNamara, A. L. Garcia, and B. J. Alder, J. Stat. Phys. 87, 1111 (1997).

[19] L.-S. Luo, Phys. Rev. E 62, 4982 (2000).
[20] L.-S. Luo and S. S. Girimaji, Phys. Rev. E 67, 036302 (2003).

[21] M. R. Swift, E. Orlandini, W. R. Osborn, and J. M. Yeomans, Phys. Rev. E 54, 5041 (1996).

[22] P. Ahlrichs, Ph.D. thesis, Johannes Gutenberg-Universität, Mainz, 2000 (unpublished).

[23] G. R. McNamara and G. Zanetti, Phys. Rev. Lett. 61, 2332 (1988).

[24] U. Frisch, B. Hasslacher, and Y. Pomeau, Phys. Rev. Lett. 56, 1505 (1986).

[25] U. Frisch, D. d'Humières, B. Hasslacher, P. Lallemand, Y. Pomeau, and J.-P. Rivet, Complex Syst. 1, 649 (1987).

[26] B. Chun and A. J. C. Ladd, Phys. Rev. E 75, 066705 (2007).

[27] M. Hénon, Complex Syst. 1, 763 (1987).

[28] I. Ginzburg and D. d'Humières, Phys. Rev. E 68, 066614 (2003).

[29] S. Chandrasekar, Rev. Mod. Phys. 15, 1 (1943).

[30] H. Risken, The Fokker-Planck Equation. Methods of Solution and Applications (Springer-Verlag, Berlin, 1984).

[31] M. Junk, A. Klar, and L.-S. Luo, J. Comput. Phys. 210, 676 (2005)

[32] E. J. Hinch, Perturbation Methods (Cambridge University Press, Cambridge, England, 1991).

[33] P. Ahlrichs and B. Dünweg, J. Chem. Phys. 111, 8225 (1999).

[34] O. Berk Usta, A. J. C. Ladd, and J. E. Butler, J. Chem. Phys. 122, 094902 (2005). 